\title{
The next step: Simplifying DAM
}

\begin{abstract}
Jennifer Neumann
is a co-founder of The Final Candidate, a new venture focused on architecting content solutions. Respected as a DAM pioneer and matter expert, global entrepreneur, market influencer and visionary, she has spoken worldwide at numerous industry events, and has frequently been interviewed by leading magazines. She holds a computer science degree from the Technical University in Berlin.
\end{abstract}

\section{Keywords: workflow, BPM, ECM, metadata, microsoft, SharePoint}

Abstract After almost 20 years, DAM still awaits its mass-market adoption. Two factors are now challenging the stand-alone DAM business: the enterprise content management (ECM) approach to orchestrate the data from all business applications, and Microsoft's recent SharePoint release in particular. DAM systems adopting the likely success of ECM might finally see broader market penetration and stand the chance to become a core component of the overall Enterprise IT Infrastructure. This paper looks at the impact of SharePoint on DAM and the resulting opportunities. The workflow technology SharePoint offers and the capability to efficiently integrate DAM with major line of business applications, or other specific technology components, indicate vast potential to increase the effect of DAM. DAM business is likely to change and customers might soon be able to select DAM components instead of locking themselves into the corner of a single DAM vendor. Journal of Digital Asset Management (2007) 3, 150-163. doi:10.1057/palgrave.dam.3650078

Jennifer Neumann The Final Candidate Fz-LLC, Dubai Internet City, Bldg.\#1, Office \#4, PO BOX 502545 UAE. Tel: +1 (415) 2873744 E-mail: jneumann@ finalcandidate.com

\section{THE NEXT STEP: SIMPLIFYING DAM}

DAM started simple: a thumbnail and a few metadata fields for managing images and a layout file for print production were enough to get traction with the first customers. Customer adoption across markets with diverse demand, however, led to an increase in the complexity involved in deploying and using DAM.

When DAM was first introduced, it was meant to take over control from the File System that was inadequate to handle assets: minimal metadata support, no thumbnails and practically no tools to preview or even convert assets were present in the File System at that time.

It all looked like an easy game for the early DAM vendors.

Almost 20 years later DAM still suffers from an insignificant share of assets being brought under control. In fact, the only real rival to the File Systems nowadays is e-mail, with most assets living as enclosures to e-mail messages.

DAM will have to continue to provide even more value and capabilities, potentially further increasing the complexity. To really succeed,
DAM solutions have to become simple again, for everyone involved.

And that is about to happen.

\section{THE PAST: DAM BUSTERS}

\section{Increasing complexity}

By definition, DAM has to deal with all kinds of assets. Initially limited to print production assets, customer demand quickly led to many kinds of assets being managed. Rich media assets, such as video, can easily come with other related asset types like still-image frames, posters, audio tracks and text, as well as the e-mail that described the project. Still today, it is predominantly images that are managed in DAM solutions.

The complexity of DAM solutions multiplied a couple of times through market impact. While rich assets initially lived almost entirely on the Macintosh and Unix servers, Microsoft managed to enter the game in the mid-1990s, adding further cross-platform complexity and increasing the problem on how and where to store metadata along with assets. 
The internet then became part of the DAM picture. Even now, no solution exists that easily serves the need for print production, web output through web content management (WCM), or even mobile phones. The concept of a digital master is great, but it is far from reality.

Every year the number of file formats increase. For example, the introduction of RAW camera files not so long ago added several dozens of still-image formats.

To most people, Metadata is just that: an alien Greek word that seems to only resonate with a few die-hard librarians. It is still quite common that customers just do not know how to use it, and many wish that someone would just force through a "standard" that would provide clear instructions on what metadata to apply, and when and by whom.

\section{The DAM islands}

In most cases, the existing DAM solutions are isolated systems allowing access to production material. DAM has not yet become a standard backbone to other systems like WCM. Instead, the vendors of those solutions have in most cases built their own, typically very limited, DAM functionality into their products.

\section{Rich media ...is for rich people only?}

The leading client/server DAM systems in the DAM market today cost tens of thousands of dollars, and some easily "scale up" to even sixdigit figures. Additional customization often easily doubles that price tag. For a single point solution, that is simply too much. Hence, customers often seek to leverage their existing hardware and software solutions, like databases, to also manage DAM assets.

\section{DAM short on creative needs}

Probably the biggest failure DAM has seen is that it has not been adopted widely by the creative world, with the exception of the central library of production assets. Even Adobe with its work in progress (WiP) DAM approach has not yet been able to make DAM an integral part of the creative process.

Take Adobe Version Cue as an example: version control should be a no-brainer functionality to help manage the drafts in a creative process, yet most users still perform "manual versioning." Or take metadata entry: most creative folks will ignore such work, given the option. The functionality most systems offer for metadata is cumbersome and not intuitive.

\section{IT outsider}

Except for some shining examples of DAM adoption by IT managers in the media \& entertainment (M\&E) industry, most companies and government organizations still do not even know what DAM stands for. Right now, they are still putting tenders out for general "e-archives."

DAM solutions frequently require a scalable architecture, either because the assets are demanding (like video) or because there are huge amounts of assets (easily tens of thousands, or more), and metadata alone can be a significant database challenge as well. There is no other way than to have IT become one of the central sponsors and supporters of DAM, providing the scalable server farm for DAM to run on.

As an industry event comparison: the first European Microsoft SharePoint Conference in Berlin, Germany, recently sold out at 2,400 mostly IT — attendees. No DAM event has yet come close to that.

\section{Integration effort too costly to maintain}

DAM serves as a platform for any functionality that is applied to the assets and metadata: rendering, search technology, digital rights management (DRM) and many more. Ideally, DAM also needs to integrate well with line of business (LoB) applications like product information systems (PIM), customer relation management systems (CRM) and others. There is usually a lot of integration involved when deploying DAM.

Shopping for a DAM solution and integrating it into the Enterprise IT infrastructure unfortunately — is not like buying a HiFi system, where you can easily pick the best receiver, amplifier or loudspeakers that fit your budget. Today's DAM systems are not built as pluggable components, and even though most DAM systems have application programming interfaces (APIs) that allow developers to integrate the DAM functionality with other systems, such integration work is usually 
expensive. What is worse, when one of the systems involved is upgraded, the integration often breaks.

The integration that DAM products offer out of the box - typically for the dominant software tools in the market - might fit the needs of a customer. If not, they are often limited and practically inextensible.

It is simply too expensive for any vendor to keep investing in all kinds of integration with the various storage, search, rendering or other technology that the large number of specialty vendors offer.

\section{THE FUTURE: DAM BOOSTERS}

So what is going to lead to better DAM adoption? Here are a few suggestions:

- leverage existing IT investments;

- centralize and simplify setup exclusively on the server;

- provide an intuitive UI that provides guidance on what tasks have to be done, and what metadata has to be entered and

- provide transparency and history for all processes, so management becomes easy.

Microsoft has recently released a new generation of SharePoint which perfectly serves to explain the points to be made. (For those not familiar with SharePoint, a few links are provided at the end of this paper, which give a good overview of what SharePoint is). ${ }^{1}$

\section{Simplicity}

Sometimes it is hard to make things easy, but providing the right tools or platform to work on makes the difference. SharePoint covers a lot of ground by setting standards on how to deploy and maintain a system, which DAM can perfectly benefit from. A few examples follow.

\section{Leverage!}

Security is an important central issue. Setting up user-access privileges is usually a challenge, especially when you have more than a couple of users. Large companies are known to spend millions of dollars to set up their Active Directory or LDAP-based permissions, and other access functionality like Single Sign-on. For the content administrators, tying DAM into
SharePoint reduces their efforts down to making simple decisions on which role or user can access which library or individual asset.

Maintenance should be handled centrally. Would not it be nice to have your assets backed up with a single click on a checkbox, along with all the other content that is stored in your SharePoint server farm? Of course IT can choose to set it up with more detail in your central Backup schedule. Or, how about functionality that allows the content administrators to use reports to analyze which assets have been previewed or used, or not?

Scalability is a must for mission-critical systems. Anyone who has ever set up a fail-over, load-balanced or clustered server solution knows about its benefits, and also that it costs significant time and money. If a company has already made that investment, for example, for a SharePoint solution, it is absolutely worth considering to tie into that scalable architecture with DAM (see Figure 1).

Microsoft builds a lot on Standards with the latest SharePoint release: web services to facilitate communication between processes, support for the BPEL workflow standard or WebDAV for version control are just a few examples. Microsoft is also committed to supporting other major browsers, Firefox and Safari, which will be crucial for its success in the creative world.

\section{User experience}

The User Experience in SharePoint is actually quite good for a web interface, but it is not great. Much can still be added to enhance it, but what is important at a fundamental level is that all UIs in SharePoint, for user and administrators alike, are implemented very consistently, which significantly reduces the training effort. Anyone who has used SharePoint before should be able to easily search and browse the asset libraries.

Users benefit from personalized content and views. SharePoint makes it easy to (self-) configure views to include certain metadata, filter the right assets and selectively provide the functionality that makes sense (see Figure 2).

Just like many other software companies, Microsoft also released a set of AJAX tools. ${ }^{2}$ Between that, and the very powerful Windows Presentation Foundation (WPF), recently introduced in .NET 3, it should be easy for system integrators (SI) and independent software 


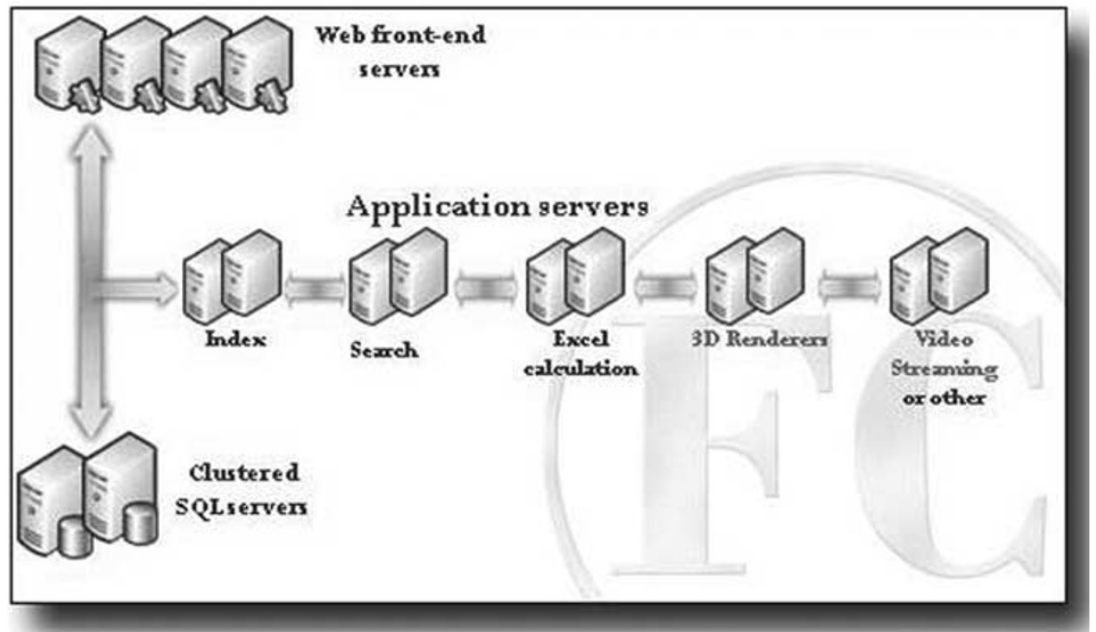

Figure 1: Sharepoint scalable architecture

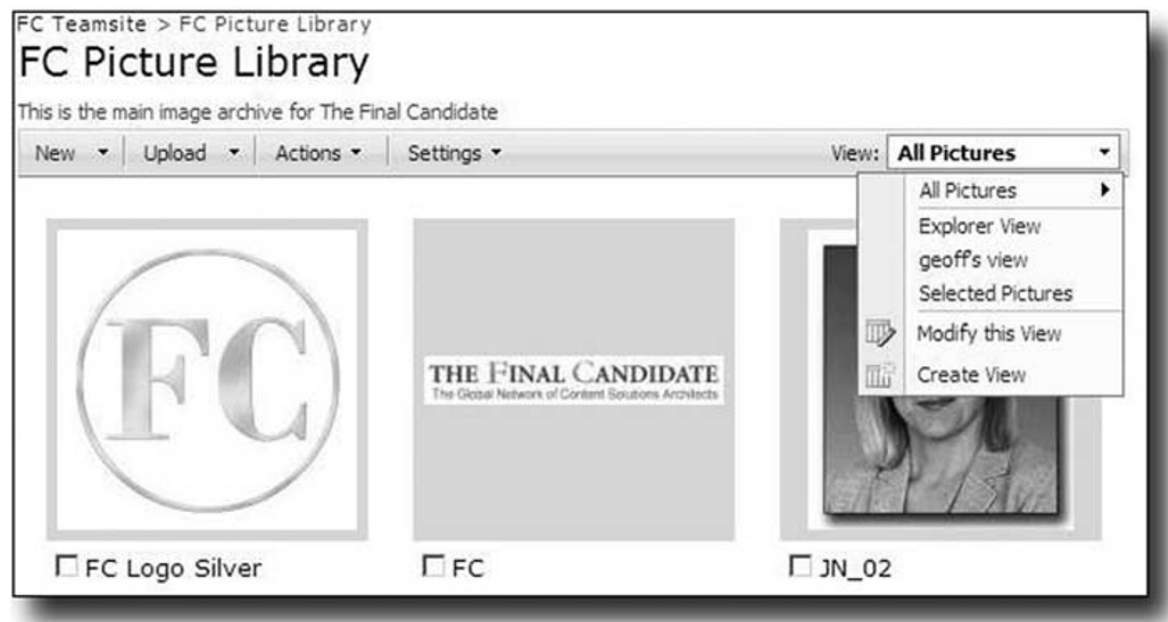

Figure 2: Metadata view setup

vendors (ISV) to rapidly produce a compelling user experience with the solutions they provide.

\section{User guidance}

Metadata entry often has been left to the gatekeeper of the DAM library. But the bulk of valuable information on assets often resides with the project managers, customers and creative staff, or even with the legal department, who are involved in creating these assets in the first place. SharePoint along with Office 2007 puts metadata on stage and into the limelight for those users who participate in the creative process (see Figure 3). Dealing with metadata requirements finally becomes a natural step for the creative user.
Within a minute, a content administrator can set up a library (eg a slide library) to include custom metadata fields, of which some can be made mandatory. PowerPoint then displays these fields right above the slide, as seen in Figure 3. If mandatory fields are not filled in, the users will not be able to check in their work, preventing the inconsistency of having assets without the necessary metadata.

SharePoint and the Office 2007 applications also tell the user what needs to be done. Microsoft has recently released the new Windows Workflow Foundation (WF) and immediately integrated it with SharePoint and Office 2007. If a user needs to act on a task, the 


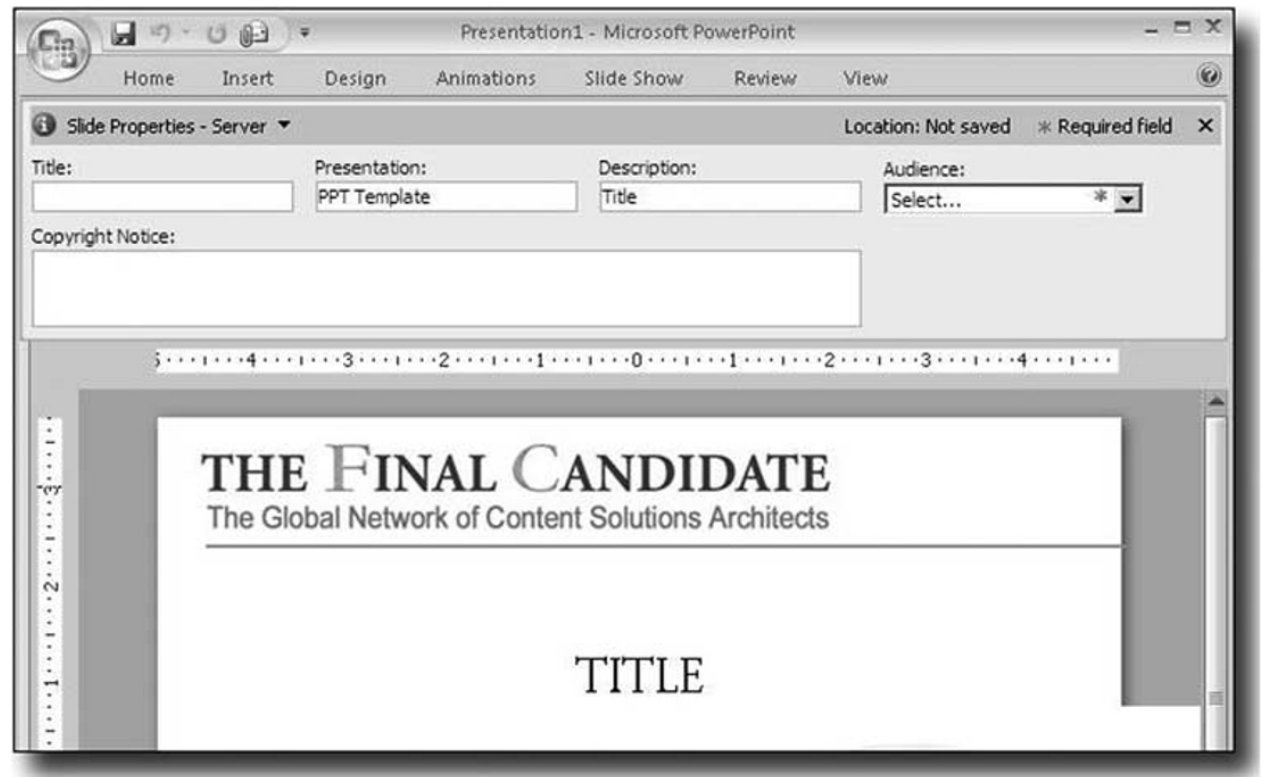

Figure 3: Metadata in PowerPoint 2007

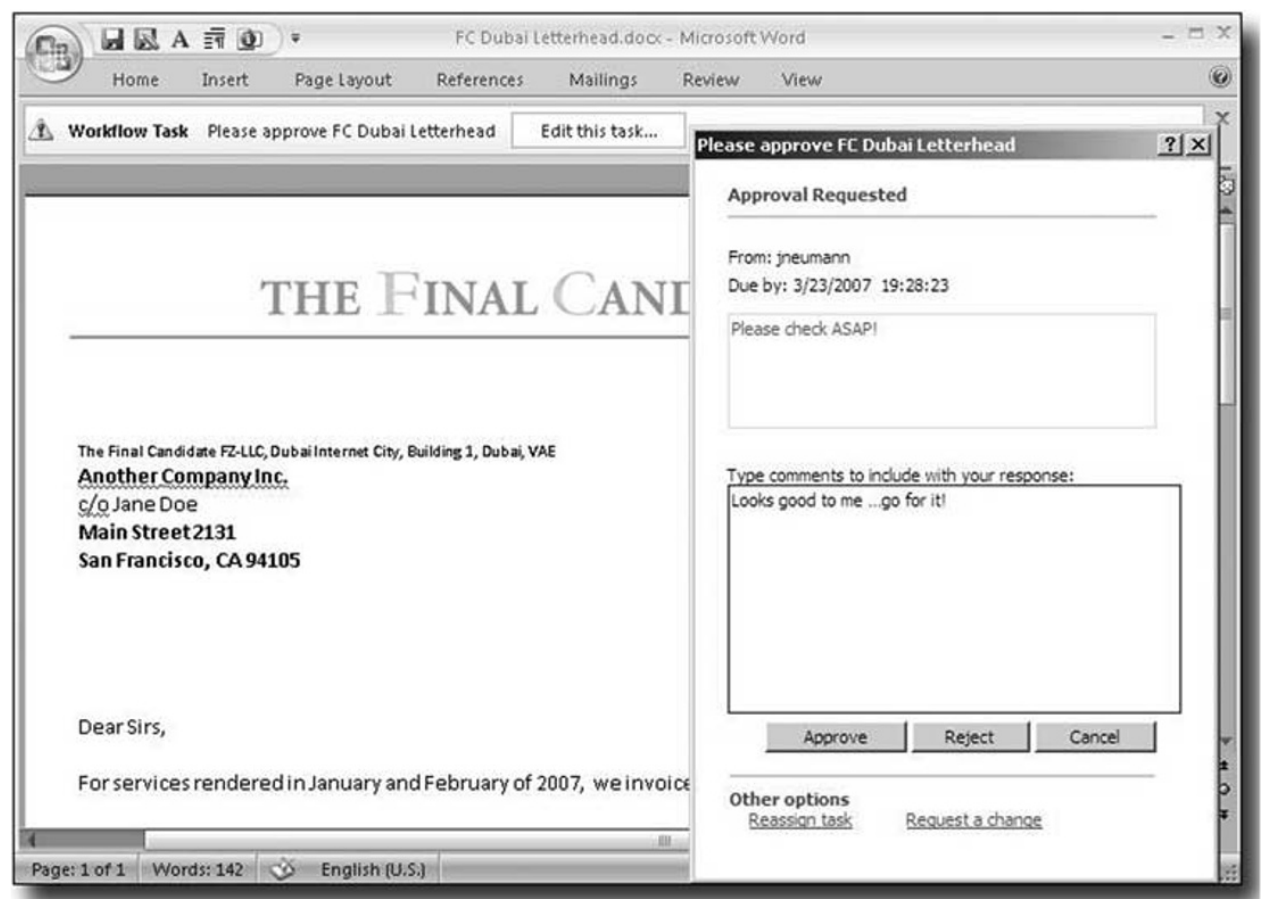

Figure 4: Task access in Word 2007

system can send an e-mail or text message notification, and the web UI as well as the Office applications will also visualize what needs to be done.
With Word 2007 users can see, in the taskbar above the document, if they have to perform a workflow task on any document that they have opened (See Figure 4). 


\section{Transparency}

Things usually become much easier if the system provides Management with the transparency and control to ensure the operation runs smoothly. SharePoint provides plenty of reporting functionality out of the box. Equally, users and administrators are given access to overviews of all the tasks currently running, or assigned to them.

Information Management Policies allow individual control (per content type) over when assets should expire, and audit settings perfectly tie in with access permissions to create trails of who accessed what.

Custom statistics can also be easily configured to visualize Audit Reports (see Figure 5).

\section{The building blocks}

As a stand-alone server solution, DAM systems need to provide all the basic components such as database access and search functionality,

\begin{tabular}{|c|c|}
\hline \multicolumn{2}{|c|}{ Run a custom report - Customize } \\
\hline \multirow{4}{*}{$\begin{array}{l}\text { Location } \\
\text { Specify whether this report } \\
\text { should be restricted to a } \\
\text { particular list in this Site } \\
\text { Collection. }\end{array}$} & \multirow{2}{*}{$\sqrt{ }$ Restrict this report to: } \\
\hline & \\
\hline & Web: All Webs \\
\hline & List: All Lists \\
\hline \multicolumn{2}{|l|}{ Date Range } \\
\hline \multirow{2}{*}{$\begin{array}{l}\text { Specify a start date and/or } \\
\text { end date that this report } \\
\text { should be restricted to. If } \\
\text { you specify only a start date, } \\
\text { the report will include all } \\
\text { events that occur after that } \\
\text { date. If you specify only an } \\
\text { end date, the report will } \\
\text { include all events that occur } \\
\text { before that date. }\end{array}$} & Start Date: \\
\hline & End Date: \\
\hline \multicolumn{2}{|l|}{$\begin{array}{l}\text { Users } \\
\text { Specify which user this report }\end{array}$} \\
\hline \multicolumn{2}{|l|}{$\begin{array}{l}\text { Specify which user this report } \\
\text { should be restricted to. }\end{array}$} \\
\hline \multirow{7}{*}{$\begin{array}{l}\text { Events } \\
\text { Specify whether this report } \\
\text { should be restricted to } \\
\text { particular events. If no event } \\
\text { filters are specified, the } \\
\text { report will include all events } \\
\text { matching the other } \\
\text { restrictions. }\end{array}$} & \multirow{2}{*}{$\begin{array}{l}\text { Opening or downloading documents, vie: } \\
\text { viewing item properties }\end{array}$} \\
\hline & \\
\hline & $\Gamma$ Editing items \\
\hline & \multirow{2}{*}{$\begin{array}{l}\Gamma \text { Checking out or checking in items } \\
\Gamma \text { Moving or copying items to another locat }\end{array}$} \\
\hline & \\
\hline & $\Gamma$ Deleting or restoring items \\
\hline & $\Gamma$ Editing content types and columns \\
\hline
\end{tabular}

Figure 5: Custom audit report 
version control and metadata management. A lot of wheels have been reinvented along the way. If DAM is deployed as part of an overall enterprise content management (ECM) approach, all these elements can and should be leveraged from the underlying platform.

Rather than integrating with each and every DAM system directly, the typical add-on technologies to DAM systems, such as watermarking or custom search technology, can be better implemented into an underlying ECM platform (like SharePoint), with direct access to the assets and the metadata.

In turn, the DAM functionality can best be accessed by other ECM components and LoB applications through a service-oriented architecture (SOA), instead of directly hardwiring separate point solutions. Of course, this is easier said than done. Pragmatically two options exist these days: either the portal (in this case SharePoint) provides base-level integration and connectors, or users consider integration as a service (IaaS), to avoid the costly point-to-point integration between separate systems.

Here is a brief look at some of SharePoint's fundamental building blocks that are essential to DAM.

\section{A common database}

SQL Server is certainly not part of the current database hype, but if you look at what a database needs to deliver to support DAM, SQL Server does a pretty good job. Decent search functionality, scalability and reliability make it a good base upon which to build DAM solutions. Plus, when it comes to databases, conservatively leaning towards "industrial strength" rather than towards "hype" might just be the right choice anyway.

Search in SharePoint goes even further: beyond the underlying search technology, SharePoint allows searches across all data stored in either SharePoint or the LoBs accessible through SharePoint. Searching for assets no longer means using a separate system.

\section{A structured approach to metadata}

Content types are a core concept in SharePoint. Content administrators can easily create new content types. As an example, it might make sense to create the content type "newswire photo" from the existing "Picture" content type. This way, all characteristics can be "inherited" from its "Parent" content type, and custom new characteristics are then added. Among the most prominent characteristics for DAM would be the particular metadata that goes with a content type. It is easy to see that newswire photos would typically come with different metadata than, for instance, photos delivered by staff photographers or agencies. By putting metadata into business context, the approach to first decide which content types should be managed helps with what metadata is required.

SharePoint also supports the Dublin Core metadata standard, as can be seen in Figure 6 .

\begin{tabular}{|lll|}
\hline Columns & & \\
\hline A column stores information about each document in the document library. Because this document library allows multiple content types, some \\
column, are now specified by the content type of the document. The following columns are currently available in this document library: \\
\hline Column (click to edit) & Type & Used in \\
Comments & Multiple lines of text & Dublin Core Columns \\
Contributor & Multiple lines of text & Dublin Core Columns \\
Coverage & Single line of text & Dublin Core Columns \\
Creator & Single line of text & Dublin Core Columns \\
Date Created & Date and Time & Dublin Core Columns \\
Date Modified & Date and Time & Dublin Core Columns \\
Date Picture Taken & Date and Time & Picture \\
Description & Multiple lines of text & Picture \\
Format & Single line of text & Dublin Core Columns \\
Keywords & Multiple lines of text & Picture, Dublin Core Columns \\
Lanouace & Choice & Dublin Core Columns \\
\hline
\end{tabular}

Figure 6: Example of metadata columns in SharePoint 
DAM vendors like Equilibrium add more metadata support, for example, for EXIF and IPTC. When you, however, look at the range of formats that vendors like iFilterShop.com add (eg support for XMP) to SharePoint, it becomes obvious that metadata support itself might soon become a customer choice. So far it was the
DAM vendors who made the choice for their customers by bundling file format utilities from vendors like Snowbound into their systems.

A standard version control system There might be more powerful version control systems in the market, but SharePoint greatly

\section{Require content approval for submitted items?}
(c) Yes
C No

Create a version each time you edit a file in this document library?

C No versioning

C Create major versions

Example: 1, 2, 3, 4

(- Create major and minor (draft) versions

Example: 1.0, 1.1.1.2, 2.0

Optionally limit the number of versions to retain:

Keep the following number of major versions:

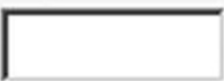

Keep drafts for the following number of major versions:

Who should see draft items in this document library?

C Any user who can read items

C Only users who can edit items

(6) Only users who can approve items (and the author of the item)

Require documents to be checked out before they can be edited?
C Yes
(c) No

Figure 7: SharePoint version control 
benefits from the built-in version control because it is easy to set up and use (see Figure 7), and because it is very flexible. It works just fine for web pages, as well as for images or slides, and it ties in nicely with the approval workflow, offering minor version (drafts) and major version (for public access).

Most importantly, the SharePoint version control system is based on WebDAV, which means that it does not lock the assets into a proprietary system, making them hard to get to. Instead, access to assets is as simple as using a URL.

Microsoft cites a size limitation of $50 \mathrm{MB}$ for assets to be stored in SharePoint. For the majority of assets, this should be no problem. For larger assets, such as video or large images for print, it means that assets should be stored outside of SharePoint, but the metadata can still be referenced through SharePoint.

\section{Business data catalog and LoBs}

The business data catalog (BDC) capability in SharePoint is of strategic importance: no portal would be complete without the ability to access the data from the LoBs, and users have come to understand that integration between these different data-centric solutions is costly.

Microsoft is providing a pragmatic approach, by not only providing interfaces (see Figure 8) but also existing integration with some of the leading LoB applications, for example, from Siebel, SAP and Oracle. Plus, the interface is made so simple that adding integration with other systems might be a matter of clicking instead of coding, by using a new product called BDC Meta Man, which promises just that.

Metadata stored in the DAM system is crucial. Variable data solutions really make sense when merging assets with data from the LoBs — like CRM or PIM — for a personalized portal.

\section{WF}

The all new WF is probably the most strategic improvement for SharePoint. While previous SharePoint versions already claimed to deliver

\section{BUSINESS DATA CATALOG (BDC) CONNECTS OFFICE SERVERS TO BUSINESS DATA}

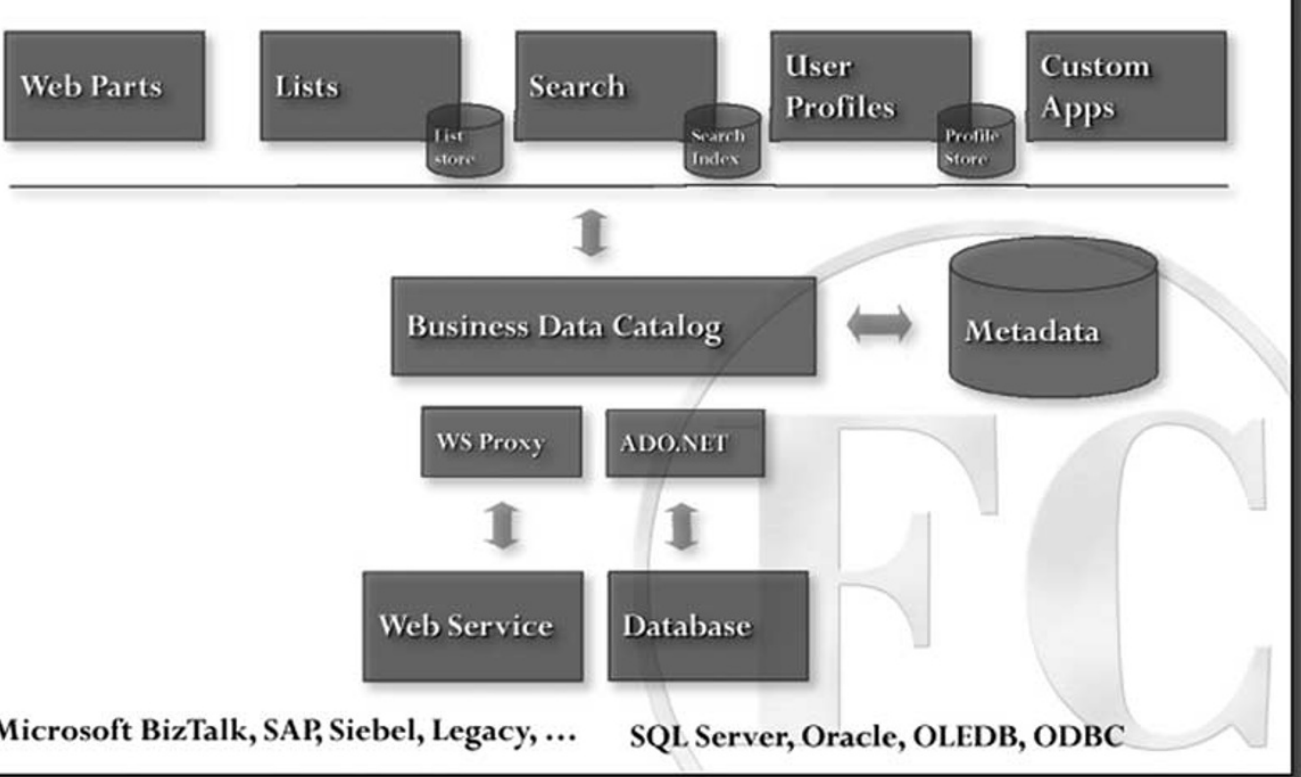

Figure 8: BDC architecture in SharePoint 
"collaboration," it is obvious that in a corporate environment reliable collaboration can only be achieved with the help of a workflow engine and business process management (BPM).

While the WF is not a BPM solution, it is a perfect foundation to build a BPM or other workflow-related solutions. Beyond the many basic activities that the WF offers, there is also a set of activities that leverage web services (see Figure 9). Via web services, workflows can control other systems, but those can also influence the execution of the workflow itself. Even better, just by invoking a menu command, an entire workflow designed in Visual Studio can be "published" as a web service.

The new Office SharePoint Designer application provides an easy, wizard-based UI to quickly compose a workflow template that automates recurring tasks. The Workflow Designer add-on to Visual Studio provides an excellent design, programming and debugging environment to build long-running, persistent workflow templates and custom activities (see Figure 10).

Any of the workflow templates installed on a SharePoint server (like the Approval workflow template that comes with SharePoint) is simply associated by a content administrator with a list or a library, and can then be instantiated on the individual list entries or assets in a library.

In the future, DAM systems will be driven by proper and general workflow solutions. When compared to that, the currently common approach to drive workflow by building triggers and scripted actions into a DAM system looks as limited as it is.

\section{Benefits}

No major adoption happens unless there are major benefits for customers. Out of the many that SharePoint offers, here is a short list of benefits that matter for DAM users.

\section{Beyond the print production ...DAM goes mobile!}

User experience is high on the list of customers these days. New ways to reach customers and innovative approaches to touch the senses of the consumers are what sells. As an example, visually rich tools like Ventuz create a lot of traction.

SharePoint itself does not offer such an exciting experience, but even the most exciting content only reaches the consumer if placed on a portal that makes it easy to find, or even pushes it towards the right target customers. Just imagine how much quicker approvals might go through if they could also be served on the new slick iPhone from Apple.

SharePoint takes good care of the nitty-gritty stuff and that is what really makes any solution work. In this scenario, it would be about making it easy for content administrators to set up "Mobile" views of the library, the assets and the workflow tasks (see Figure 11).

\section{Ease of use}

Ease of Use is required for all business aspects: administration, creative and business, and it starts with not having to learn yet another system, but to have all systems sharing common concepts to configure and access their data. That is one of the main advantages that the ECM approach has.

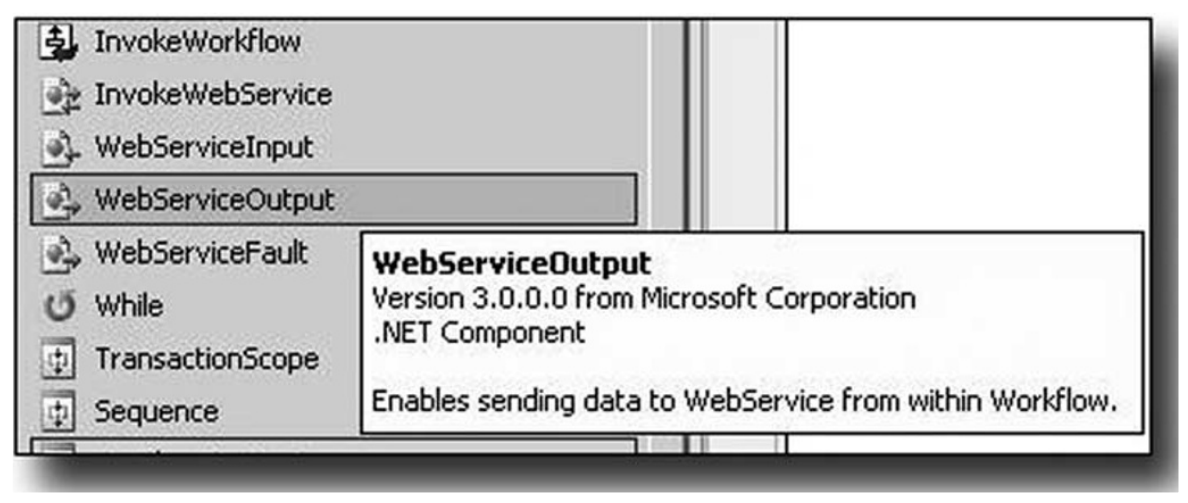

Figure 9: Web services workflow activities 


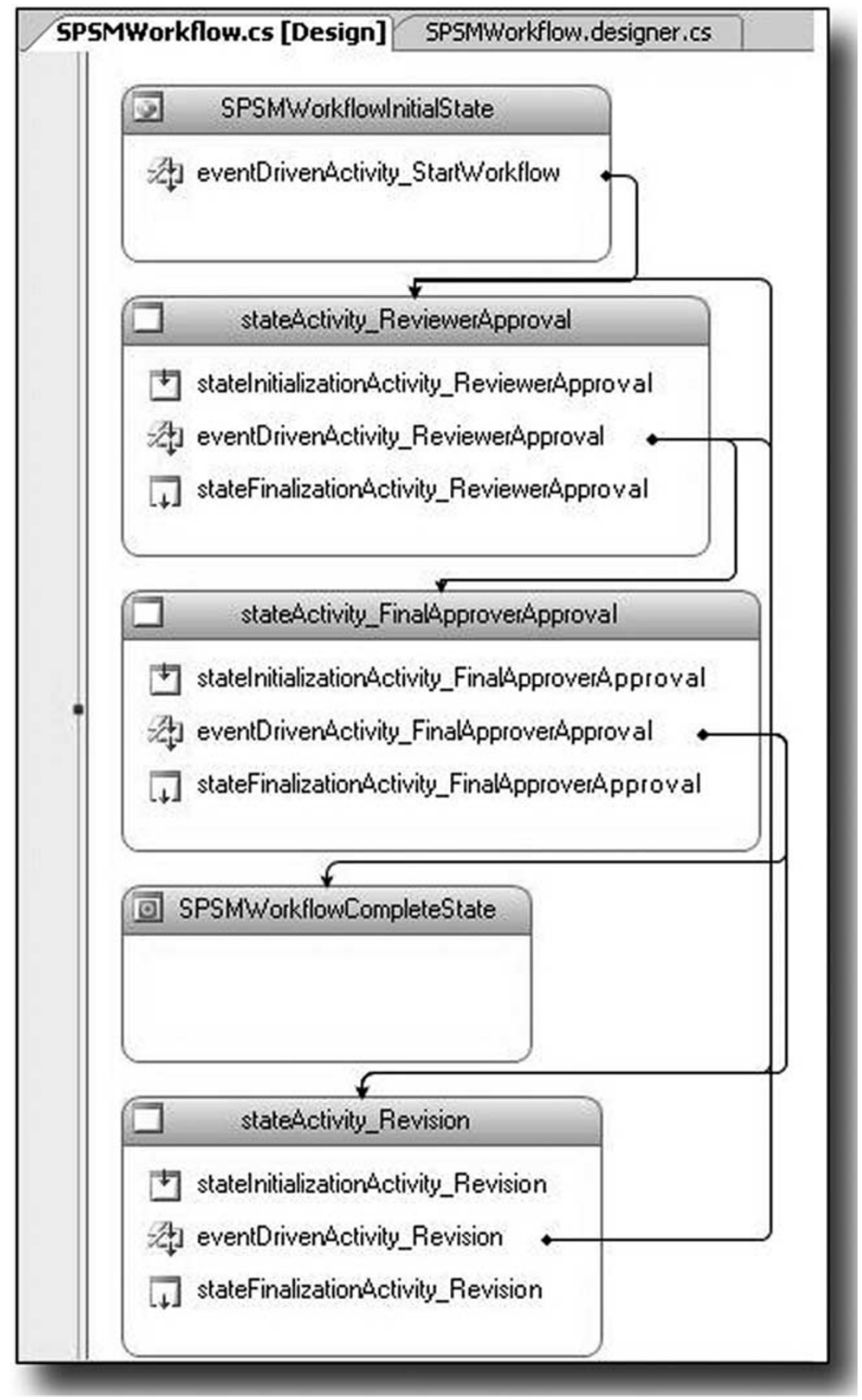

Figure 10: Multiple approvers workflow

It will be interesting to see how creative users will react to a SharePoint-based DAM solution. Wherever SharePoint has already been installed and DAM was added to the mix, it usually has not been an issue and with corporate assets such as sales presentations created in PowerPoint 2007 , it is safe to predict that users will be quite happy.

For other areas like the print world or video production, the jury is still out on whether 


\begin{tabular}{|c|c|}
\hline$\boxplus$ Group By & \\
\hline \multicolumn{2}{|l|}{$\boxplus$ Totals } \\
\hline \multicolumn{2}{|l|}{$\boxplus$ Style } \\
\hline \multicolumn{2}{|l|}{$\boxplus$ Folders } \\
\hline \multicolumn{2}{|l|}{$\boxplus$ Item Limit } \\
\hline \multicolumn{2}{|l|}{$\exists$ Mobile } \\
\hline \multirow[t]{3}{*}{ Adjust mobile settings for this view. } & $\begin{array}{l}\text { Make this a mobile view } \\
\text { (Applies to public views only) }\end{array}$ \\
\hline & $\begin{array}{l}\text { Make this the default mobile view } \\
\text { (Applies to public views only) }\end{array}$ \\
\hline & $\begin{array}{l}\text { Web address for this mobile view: } \\
\text { http://www. finalcandidate.com: } 9970 / \text { lay } \\
\text { 2Dc931\%2D } 4 \text { d } 52 \% 209 c 01 \% 20 c 9377 \text { fob } \\
\text { 2D } 9872 \% 205 a a 041 a 0 a 266 \\
\text { Web address for mobile home page: } \\
\text { http://www. finalcandidate.com: } 9970 / \text { lay }\end{array}$ \\
\hline
\end{tabular}

Figure 11: Mobile view setup in SharePoint

SharePoint will become relevant. Several pieces of the puzzle still have to be added. Here too, process is an important subject for any business, so any vertical market solution addressing the special needs of those users and offering seamlessly integrated workflow functionality, as achieved with Office 2007, will likely win over a significant share of the niche market.

Ease of Use is certainly much easier to achieve when building a solution with a clear target customer need in mind. The question is how quickly vertical market solutions, and the components providing market-specific functionality to build them, will come along. For noncreative markets like Legal, this has already happened.

\section{Measurable ROI and efficiency gains}

Workflow holds the promise to substantially increase efficiency. Just the fact that with the right workflow processes in place automation increases and manual work is less likely to be forgotten creates expectations for vast improvements. But along the lines of "If you can't measure it, you can't manage it," it is equally important to recognize that by using a workflow engine performance can be measured and inefficiencies can be identified. With the transparency provided by the workflow statistics, it will finally become easier to justify the use of DAM and to determine its measurable ROI. ${ }^{3}$

\section{Risk management}

Compliance has been repeatedly quoted as a major driver for adoption. If you are subject to scrutiny like SOX, using SharePoint might cover your back with its records management functionality.

What most people have not yet recognized is that basic records management functionality, such as audits or scheduling, also makes total sense for assets.

\section{Changing business models}

SharePoint will have a major impact on customers, vendors, SIs and even analysts. On the blogs and in discussions groups some have already started calling it the new (higher-level) OS. This will cause many changes to existing business models. ${ }^{4}$ 
Customers who deploy such an ECM platform can expect that adding another solution will be an add-on incremental step, with staged deployments, instead of yet another parallel complete learning and testing cycle.

For DAM vendors there are many opportunities: for the first time it will be possible to sell point functionality as SharePoint components. A specialty vendor might focus and excel on just one type of asset, such as video, and still succeed. Others might still aim at building the "complete" DAM platform, but at least they can stop reinventing wheels, like version control or databases.

SharePoint is a significant hurdle for SIs. It is quite encompassing, and most SIs simply cannot afford to learn and evaluate SharePoint for three months to find out what they could do with it. As an example, however, DPCI one of the leading Publishing SIs out of New York - has already been an early adopter of SharePoint. Chances are that others will follow soon. ${ }^{5}$

ECM even creates a challenge for analysts. No longer can they specialize in just one area, but at the same time their knowledge needs to go into some detail to be able to judge and compare. Detailed case studies, combining vertical market and process management expertise, will be in high demand!

\section{OTHER ALTERNATIVES ...WHERE ART THOU?}

While most seem to have looked at the Vista release (which, by the way, also has a few nice features and screen real estate for metadata), the latest Big Bang from Microsoft was really SharePoint. Slowly but surely, people are starting to understand that.

The question now is: what is the competition up to?

\section{Open source}

The open source world actually has all the components that it would take to build a similar platform. Is anyone working on that? Who would take central responsibility to maintain any tight integration between these core components?

\section{Database, OS, Portal Vendors ... anybody?}

Analysts have often predicted that the major database vendors would be pushing forward in this direction. Documentum has already had good experience and revenues with ECM.

A feature, which typically only strategic marketing experts appreciate, might just work out well for Microsoft and SharePoint here: pricing. Oracle and Documentum are presently too highly priced to find the mass-market adoption that Microsoft can now hope for.

Apple seems to be more focused on the consumer market than the server. Sun seems to have lost the momentum from the success the Java platform gave them.

Apple did not seem to understand the strategic importance of the server when they allowed Microsoft in the 1990s to take over the server business in the Publishing world, with something as lame Windows NT. In this regard, 2007 might become a groundhog year for Apple.

\section{laaS}

If your main focus these days is on LoB integration, some of the "up and coming" IaaS start-ups have received great feedback from the market and are worth considering. Despite the fact that they still have to prove themselves, and are yet to mature and to scale their businesses, they might be worth investing in trial projects.

As a basis for DAM, they are, however, not an alternative to a platform like SharePoint.

\section{OUTLOOK}

Undoubtedly, SharePoint will have a major impact. Here are a few specific predictions:

Technology gravity strikes again: The Windows SharePoint Services (WSS v3), which is the basis of the various commercial SharePoint products, comes for free - except of course for the Windows Server 2003 license it runs on. It includes all the core platform components such as the database, workflow and version control, as well as an image library. This puts vendors of stand-alone DAM products for workgroups in severe jeopardy.

Workflow will become mainstream: Scripted and "hot folder" — based solutions are ultimately inferior and will lose importance.

SharePoint adoption will take a bit of time: InfoTrends predicts that it will happen much 
quicker than many other technology adoption cycles, with major success at the "workgrouplevel" and even initial enterprise deployments this year! ${ }^{6}$

DAM business will change significantly: With SharePoint handling the basics, DAM vendors can focus on the heavy lifting. Managing large images demands server-side previews and annotation capabilities. High-quality conversion of color images in batches, or streaming video. Cool search technology, rights management, or secure and performing file transfers to clients. All these can be delivered by specialty vendors delivering separate components, and content architects can take these components and build DAM solutions for vertical markets or special applications.

Microsoft secures its lead in server market share: Even more importantly, Microsoft extends its dominance towards the desktop and will strongly influence the user experience. Two years ago, a leading analyst stated that ECM is a myth. Today, ECM has become something that is within realistic reach for many.

\section{References}

1 InfoWorld. Opinion: Is Microsoft's SharePoint 2007 a golden app? http://www.computerworld. $\mathrm{com} /$ action/article.do?command=viewArticleBasic \&articleId=9008561\&source $=$ rss_news 50 .

2 MS SharePoint Links.

- SharePoint Homepage: http://www.microsoft. com/sharepoint

- SharePoint version comparison: http://office. microsoft.com/en-us/sharepointserver/ FX101758691033.aspx

- SharePoint AJAX Homepage: http://ajax.asp. net/\#\#http://www.codeplex.com/ sharepointajax/SourceControl/ ListDownloadableCommits.aspx

- BDC Meta Man product: www.bdcmetaman.com.

3 Neumann, J., Major efficiency gains will come from mainstream workflow applications. http:// www.finalcandidate.com/en/tandp/Pages/ SharePointWorkflow.aspx.

4 Davis, Z., SharePoint: The next big 'operating system' from Microsoft? http://blogs.zdnet.com/ microsoft $/$ ? $\mathrm{p}=327$.

5 DPCI. http://www.databasepublish.com/.

6 InfoTrends. http://www.infotrends.com/public/ Content/Services/DSS/dss.html. 